\title{
Orally administered simvastatin partially preserves lumbar vertebral bone mass but not integrity of intervertebral discs in ovariectomized rats
}

\author{
FA-MING TIAN $^{1 *}$, SHU-YANG LI ${ }^{2 *}$, KAI YANG $^{2}$, YANG LUO $^{2}$, \\ MU-WEI DAI ${ }^{2}$, GUANG-YUAN LIU ${ }^{2}$, HUI-PING SONG ${ }^{2}$ and LIU ZHANG ${ }^{2}$ \\ ${ }^{1}$ Medical Research Center, North China University of Science and Technology; ${ }^{2}$ Department of Orthopedic Surgery, \\ Affiliated Hospital of North China University of Science and Technology, Tangshan, Hebei 063000, P.R. China
}

Received October 13, 2015; Accepted November 10, 2016

DOI: 10.3892/etm.2017.4043

\begin{abstract}
The present study aimed to investigate the effect of orally administered simvastatin on lumbar vertebral bone mass and intervertebral disc (IVD) degeneration in ovariectomized (OVX) rats. A total of 30 female Sprague-Dawley (SD) rats were subjected to either bilateral ovariectomy $(n=20)$ or sham surgery $(n=10)$. After 12 weeks, the OVX rats were orally administered either saline vehicle (OVX $+\mathrm{V}$ group; $\mathrm{n}=10)$, or $5 \mathrm{mg} / \mathrm{kg} /$ day simvastatin (OVX + SIM group; $\mathrm{n}=10$ ). Following 12 weeks of treatment, necropsy was conducted and bone mineral density (BMD) was determined in the L5-6 vertebrae. Furthermore, the microstructure and biomechanical properties of the L3 vertebrae were detected by micro-computed tomography and compression testing, respectively. The L5-6 vertebrae were analyzed by measurement of IVD height, observation of histological changes by van Gieson staining, and evaluation of collagen-II (col-II), aggrecan (AGG) and collagen I (col-I) expression by immunohistochemical analysis. Rats in the OVX+V group had lower BMD, bone volume/trabecular volume ratio, maximum load and elastic modulus than the sham group. Rats in the OVX + SIM group had higher BMD and biomechanical strength values than the rats in the $\mathrm{OVX}+\mathrm{V}$ group. Histological analysis showed that the $\mathrm{OVX}+\mathrm{V}$ and OVX + SIM groups exhibited significantly higher disc degeneration scores and significantly lower IVD height than the sham group. Immunohistochemical analysis revealed lower expression levels of col-II and AGG, but higher levels of col-I
\end{abstract}

Correspondence to: Dr Liu Zhang, Department of Orthopedic Surgery, Affiliated Hospital of North China University of Science and Technology, 73 Jianshe South Road, Tangshan, Hebei 063000, P.R. China

E-mail: zhliu130@sohu.com

*Contributed equally

Key words: simvastatin, bone mineral density, intervertebral disc in the annulus fibrosis and endplate in $\mathrm{OVX}+\mathrm{V}$ rats compared with the sham group. The OVX + SIM group exhibited levels of col-II, AGG and col-I expression comparable with those of $\mathrm{OVX}+\mathrm{V}$ rats, with the exception of an upregulation of col-II expression in the annulus fibrosis. These data demonstrate that simvastatin treatment partially prevented bone loss and the deterioration of biomechanical properties of lumbar vertebrae, but not the progression of IVD degeneration in OVX rats.

\section{Introduction}

Lower back pain is responsible for a large amount of human suffering, high healthcare costs and significant socioeconomic losses. Furthermore, the burden of lower back pain on the individual and society is expected to increase markedly because of the aging population (1). Clinically, a large number of patients with persistent lower back pain attribute their symptoms to intervertebral disc (IVD) degeneration (2). More than $90 \%$ of surgical spine surgeries are performed as a result of IVD degeneration (3). There is a great need for an oral prophylactic drug that is able to inhibit the pathogenesis of disc degeneration, and thereby improve the quality of life of patients and reduce the burden of lower back pain on individuals and society.

Although IVD degeneration has a multifactorial etiology, involving age, mechanical, genetic, systemic and toxic factors $(4,5)$, increasing evidence suggests an association between changes in the vertebral body and IVD degeneration. Fabreguet et al (6) demonstrated that the prevalence of lumbar spine degeneration is high in osteoporotic men. Furthermore, relative estrogen deficiency may contribute to accelerated disc degeneration in postmenopausal females (7). In addition, our previous studies revealed a strong association between osteopenia and disc degeneration in ovariectomized (OVX) rats, and intervention using an antiresorptive agent not only prevented osteopenia, but also inhibited IVD degeneration (8-10). Considering IVD degeneration most often occurs in elderly individuals, it is likely to be combined with other conditions to which the elderly are susceptible, such as osteoporosis and hyperlipidemia. It would be interesting 
if treatments for hyperlipidemia could also be effective for preventing osteoporosis and IVD degeneration.

Intriguingly, a 3-hydroxy-3-methylglutaryl coenzyme A (HMG-CoA) reductase inhibitor, simvastatin, commonly prescribed as a cholesterol-lowering drug, has been reported to not only promote bone formation in both clinical and experimental studies (11-15), but also slow IVD degeneration in a rat model by intradisc injection (16). However, to date, it remains unknown whether orally administered simvastatin is able to inhibit the pathological process of IVD degeneration.

Therefore, the aim of the present study was to determine if simvastatin could prevent bone loss while also inhibiting IVD degeneration in OVX rats.

\section{Materials and methods}

Experimental design. A total of 30 female Sprague-Dawley (SD) rats (3 months old), with average weight of $260 \pm 15 \mathrm{~g}$, obtained from Peking University Animal Center (Beijing, China) were used in this study. Rats were maintained in an environment maintained at a temperature of $22 \pm 2^{\circ} \mathrm{C}$, a humidity level of 55-60\% and a 12-h light/dark cycle. Rats had access to food and water ad libitum. Of these rats, 20 rats received bilateral ovariectomy (the OVX group), and the remaining 10 rats were used as controls, in which the ovaries were exteriorized but not removed (the sham group). At week 12, the OVX rats were randomly divided into two groups and orally administered: i) saline vehicle treatment (OVX + V group; $\mathrm{n}=10)$ or ii) simvastatin (Ruibang Pharmaceutical Co., Ltd., Wenzhou, China) treatment at a dose of $5 \mathrm{mg} / \mathrm{kg} /$ day (OVX + SIM group; $\mathrm{n}=10)$. At week 24, all animals were sacrificed by cervical dislocation. All experimental procedures were approved by the Institutional Animal Care and Use Committee of North China University of Science and Technology (Tangshan, China).

Bone mineral density (BMD) assessment. For all animals, BMD of L5, L6 and L5-6 was determined on the anteroposterior plane, by dual energy X-ray absorptiometry using a densitometer (QDR Discovery; Hologic Inc., Bedford, MA, USA) operating at high-resolution mode, and specialized software (version 13.3; Hologic, Inc., Marlborough, MA, USA) for small animals supplied by the equipment manufacturer.

Micro-computed tomography (CT) measurements. Three-dimensional (3D) analysis was performed on the trabeculae of the cancellous tissue of the L3 vertebra, using a SkyScan 1076 micro-CT scanner (Bruker MicroCT, Kontich, Belgium). The micro-CT equipment comprised a microfocus X-ray tube with a focal spot of $9 \mu \mathrm{m}$ to produce a fan beam detected by a charge-coupled device array, and a turntable that could be shifted automatically in the axial direction. The energy and intensity were equal to $40 \mathrm{kVp}$ and $250 \mu \mathrm{A}$, respectively. On the original $3 \mathrm{D}$ images, morphometric indices were directly determined from the volume of interest (VOI), which was restricted to an inner cylinder with $1.5 \mathrm{~mm}$ diameter and the entire vertebra, excluding the region $1 \mathrm{~mm}$ from the proximal and distal growth plate. The following 3D morphometric parameters were calculated to describe the bone mass and microstructure: Percentage of bone volume to total volume (BV/TV), trabecular number (Tb.N), trabecular thickness (Tb.Th), trabecular separation (Tb.Sp) and structural model index (SMI).

Biomechanical testing. Using a mechanical strength analyzer (AG-IS 10kN; Shimadzu Corporation, Tokyo, Japan), the mechanical strength of the lumbar vertebra (L3) was measured by compression test (16). In this test, the planoparallel surfaces were obtained by removing the cranial and caudal ends of the vertebral specimen, thereby allowing for a uniform compression test to be performed on individual rodent vertebrae. From the vertebral body, a central cylinder with planoparallel ends and a height of $\sim 5 \mathrm{~mm}$ was obtained. All compression tests were performed in the displacement-control mode at a crosshead speed of $0.5 \mathrm{~mm} / \mathrm{min}$ to eliminate any strain rate effects. Maximum load, yield stress, maximum stress, and elastic modulus were obtained from compression tests of the vertebral bodies.

Histological and histomorphometric evaluation. The L5-6 segments of the lumbar spine (including the intervertebral disc) were fixed in neutral-buffered $10 \%$ formalin, decalcified and embedded in paraffin. Samples were then cut into $5-\mu \mathrm{m}$ sections and stained with van Gieson's stain (VG) for light microscopic examination.

Degenerative changes in the intervertebral discs were observed and the degrees of change in the stained sections were scored independently by three individuals blinded to the experimental protocol, using the disc degeneration assessment scoring system (Table I) described by Wang et al (8).

Disc height measurements were taken from the caudal aspect of the growth plate of L5 to the cranial aspect of the growth plate of L6 on histological samples from the L5-6 segments. For each image, an average of three measurements made from three areas of the disc space for one section from each rat: One from the anterior, one from the central and one from the posterior side (17). The thickness of the endplate was measured from the cranial growth plate to the border between the nucleus pulposus and the endplate in the VG staining. The ratio of calcified area to the total endplate area was also determined. All measurements were performed using a digital image analysis system (Cell Sens; version 1.8; Olympus Soft Imaging solutions GmbH, Münster, Germany).

Immunohistochemical analysis. Tissue sections $(5 \mu \mathrm{m})$ were deparaffinized in xylene and rehydrated in a reverse-graded series of ethanol. Antigen retrieval was performed using $0.1 \mathrm{M}$ sodium citrate (J\&K Scientific Ltd., Beijing, China), endogenous peroxidase activity was suppressed by $0.3 \% \mathrm{H}_{2} \mathrm{O}_{2}$ for $15 \mathrm{~min}$, and then were blocked for $30 \mathrm{~min}$ using blocking solution (TSA kit; cat no. NEL749A001KT; PerkinElmer, Inc., Waltham, MA, USA) blocking of non-specific binding, sections were incubated overnight at $4^{\circ} \mathrm{C}$ with anti-rat collagen-II (Col-II; 1:100; cat no. 79013Abcam, Cambridge, MA, USA), aggrecan (AGG) or collagen-I (Col-I) antibodies (both 1:100; cat nos. bs-11655R and bs-0578R, respectively; BIOSS, Beijing, China). The remaining procedures were performed using an SABC-FITC kit (SA1066; Wuhan Boster Biological Technology, Ltd., Wuhan, China) according to 
Table I. Scoring system for assessment of lumbar intervertebral disc degeneration.

\begin{tabular}{llll}
\hline Score & \multicolumn{1}{c}{ Nucleus pulposus } & \multicolumn{1}{c}{ Annulus fibrosus } & Osteophyte \\
\hline 0 & Bulging gel with abundant notochordal cells & Absence \\
1 & $\begin{array}{l}\text { Notochordal cell loss; chondrocyte-like cell } \\
\text { emergence }\end{array}$ & $\begin{array}{l}\text { Compact fibrous lamellae } \\
\text { Proliferation of fibrocartilaginous } \\
\text { tissue and loss of } \\
\text { nuclear-annular border } \\
\text { Appearance }\end{array}$ \\
3 & $\begin{array}{l}\text { Focal mucoid degeneration; clefts } \\
\text { Diffuse mucoid degeneration and clefts }\end{array}$ & & Fissures in annulus fibrosis \\
throughout nucleus & & - \\
\hline
\end{tabular}

Table II. Results of bone mineral density testing $\left(\mathrm{g} / \mathrm{cm}^{2}\right.$; mean \pm standard deviation, $\mathrm{n}=10$ ).

\begin{tabular}{lccc}
\hline Vertebra & Sham & OVX + V & OVX + SIM \\
\hline L5 & $0.2933 \pm 0.011$ & $0.2423 \pm 0.0081^{\mathrm{a}}$ & $0.2588 \pm 0.0173^{\mathrm{a}, \mathrm{b}}$ \\
L6 & $0.2907 \pm 0.015$ & $0.2395 \pm 0.0061^{\mathrm{a}}$ & $0.2565 \pm 0.0129^{\mathrm{a}, \mathrm{b}}$ \\
L5-6 & $0.286 \pm 0.0115$ & $0.238 \pm 0.0059^{\mathrm{a}}$ & $0.2519 \pm 0.0124^{\mathrm{a}, \mathrm{b}}$ \\
\hline
\end{tabular}

${ }^{\mathrm{a}} \mathrm{P}<0.05$ vs. sham group; ${ }^{\mathrm{b}} \mathrm{P}<0.05$ vs. OVX $+\mathrm{V}$ group. OVX, ovariectomized; V, vehicle; SIM, simvastatin.

the manufacturer's instructions, and the color (brown) was developed by incubation in DAB (ZSGB-BIO, Beijing, China). Sections were counter-stained with hematoxylin. All sections were observed by a light microscope (BX 53; Olympus Corporation, Tokyo, Japan) and semi-quantitatively analyzed using Image Pro Plus version 6.0 software (Media Cybernetics, Inc., Rockville, MD, USA) and the integrated optical density (IOD) was measured on the images at x400 magnification.

Statistical analysis. All data were analyzed using SPSS 19.0 software (IBM SPSS, Armonk, NY, USA) and results are expressed as the mean \pm standard deviation. The statistical significance of differences between groups was estimated using one-way analysis of variance and Fisher's protected least significant difference test. The results of histological scores were analyzed using a non-parametric Kruskal-Wallis test. $\mathrm{P}<0.05$ was considered to indicate a statistically significant difference.

\section{Results}

Bone mineral density of L5-6 vertebrae. As shown in Table II, the BMD of L5, L6 and L5-6 in the rats of the OVX $+\mathrm{V}$ and OVX + SIM groups were all significantly lower compared with those of the sham group. Rats of the OVX + SIM group exhibited markedly higher BMDs than those of the OVX $+\mathrm{V}$ group (Table II).

Micro-CT analysis of L3 vertebrae. Changes in the 3D microarchitectural properties of the subchondral trabecular bone were visible in the representative $3 \mathrm{D}$-reconstructed
Table III. Results of micro-computed tomography (mean \pm standard deviation, $\mathrm{n}=10$ ).

\begin{tabular}{lccc}
\hline Parameters & Sham & OVX + V & OVX + SIM \\
\hline BV/TV $(\%)$ & $23.7 \pm 4.8$ & $17.6 \pm 3.0^{\mathrm{a}}$ & $18.4 \pm 1.0^{\mathrm{a}}$ \\
Tb.Th $(\mu \mathrm{m})$ & $101.1 \pm 3.2$ & $103.0 \pm 3.1$ & $101.6 \pm 1.3$ \\
Tb.N $\left(\mathrm{mm}^{-1}\right)$ & $2.3 \pm 0.4$ & $1.7 \pm 0.3^{\mathrm{a}}$ & $1.8 \pm 0.1^{\mathrm{a}}$ \\
Tb.Sp $(\mu \mathrm{m})$ & $254.6 \pm 23.5$ & $348.2 \pm 13.3^{\mathrm{a}}$ & $326.0 \pm 16.8^{\mathrm{a}}$ \\
SMI & $1.3 \pm 0.4$ & $1.4 \pm 0.1$ & $1.3 \pm 0.1$ \\
\hline
\end{tabular}

${ }^{\mathrm{a}} \mathrm{P}<0.05$ vs. sham group. OVX, ovariectomized; V, vehicle; SIM, simvastatin; BV, bone volume; TV, total volume; Tb.Th, trabecular thickness; Tb.N, trabecular number; Tb.Sp, trabecular separation; SMI, structural model index.

micro-CT images of the vertebral trabecular bone of the VOI from each group (Fig. 1). Quantification of 3D trabecular structures revealed that in the OVX $+\mathrm{V}$ and OVX $+\mathrm{SIM}$ groups, BV/TV and Tb.N were significantly decreased $(\mathrm{P}<0.05)$ and Tb.Sp was significantly higher $(\mathrm{P}<0.05)$, compared with their values in the sham group. OVX + SIM rats exhibited higher values of $\mathrm{BV} / \mathrm{TV}$ and $\mathrm{Tb} . \mathrm{N}$, and lower values of Tb.Sp than did the OVX $+\mathrm{V}$ group, although the differences were not statistically significant (Table III).

Biomechanical properties of L3. As shown in Table IV, maximum load, yield stress, maximum stress and elastic modulus in the OVX $+\mathrm{V}$ group were significantly lower compared with those in the sham group $(\mathrm{P}<0.05)$. Furthermore, rats in the OVX + SIM group exhibited significantly higher values than those in the OVX $+\mathrm{V}$ group $(\mathrm{P}<0.05)$.

Histological and histomorphometric observations in L5-6 vertebral discs. The sham group exhibited mild degeneration, with partial calcification of the cartilage endplates and a few doublets of chondrocyte-like cells within the nucleus pulposus at the rear side. In addition to diminished disc height, the OVX $+\mathrm{V}$ and OVX + SIM groups experienced more serious degeneration of the endplate, annulus fibrosus and nucleus pulposus. Mineralized tissue was more evident in the deep zone of the middle cartilage endplate. In the nucleus pulposus, notochordal cells exhibited a significant loss of cellularity and were replaced by chondrocyte-like 
Table IV. Results of biomechanical testing (mean \pm standard deviation, $\mathrm{n}=10$ ).

\begin{tabular}{lccc}
\hline Parameters & Sham & OVX + V & OVX + SIM \\
\hline Maximum load (N) & $204.6 \pm 19.4$ & $91.1 \pm 25.6^{\mathrm{a}}$ & $184.8 \pm 52.6^{\mathrm{b}}$ \\
Elastic modulus (MPa) & $823.6 \pm 158.3$ & $563.9 \pm 277.8^{\mathrm{a}}$ & $802.3 \pm 218.1^{\mathrm{b}}$ \\
Maximum stress (MPa) & $19.7 \pm 4.5$ & $13.0 \pm 5.09^{\mathrm{a}}$ & $18.1 \pm 5.9^{\mathrm{b}}$ \\
Yield stress (MPa) & $17.7 \pm 3.7$ & $11.3 \pm 4.5^{\mathrm{a}}$ & $16.9 \pm 5.1^{\mathrm{b}}$ \\
\hline
\end{tabular}

${ }^{\mathrm{a}} \mathrm{P}<0.05$ vs. sham group; ${ }^{\mathrm{P}}<0.05$ vs. OVX + V group. OVX, ovariectomized; V, vehicle; SIM, simvastatin.

cells. Mucoid degeneration was visible, which eroded the nucleus pulposus and formed an apparent cleft. Loss of collagen, proliferation of fibrocartilaginous tissue and disruption of the nuclear-annular border were observed at the annulus fibrosus. Accordingly, the histological scores of disc degeneration in the OVX $+\mathrm{V}$ and OVX + SIM groups were significantly higher compared with the histological score in the sham group $(\mathrm{P}<0.05$; Fig. 2$)$.

Disc height in the OVX $+\mathrm{V}(1.09 \pm 0.09 \mathrm{~mm})$ and OVX + SIM (1.10 $\pm 0.08 \mathrm{~mm})$ groups was significantly lower compared with that in the sham group $(1.25 \pm 0.08 \mathrm{~mm}$; $\mathrm{P}<0.05)$. Endplate thickness in the OVX $+\mathrm{V}(198.9 \pm 32.0 \mu \mathrm{m})$ and OVX + SIM $(182.5 \pm 22.7 \mu \mathrm{m})$ groups was significantly lower compared with that in the sham group $(141.8 \pm 22.8 \mu \mathrm{m}$; $\mathrm{P}<0.05)$, and the same trends were found in the ratio of calcified area to endplate area of the endplate of L5-6 discs (33.5 \pm 5.9 and $31.7 \pm 4.8$ vs. $20.8 \pm 3.5 \%$; $\mathrm{P}<0.05$; Fig. 3 ).

Immunohistochemical staining. Immunohistochemical staining and IOD analysis showed that the protein expression of Col-II in the annulus fibrosis in the OVX $+\mathrm{V}$ group was lower than that in both the sham and OVX + SIM groups $(\mathrm{P}<0.05)$, while in the nucleus pulposus, Col-II expression was significantly higher in the OVX $+\mathrm{V}$ and OVX + SIM groups than in the sham group, while the opposite was observed in the endplate $(\mathrm{P}<0.05$; Fig. 4$)$.

The protein expression levels of $\mathrm{AGG}$ in the annulus fibrosis and endplate in the OVX $+\mathrm{V}$ and OVX + SIM groups were markedly lower than in the sham group $(\mathrm{P}<0.05)$, while there was no significant difference between any two groups in nucleus pulposus (Fig. 5).

The protein expression levels of col-I in the annulus fibrosis and endplate in the OVX $+\mathrm{V}$ and OVX + SIM groups were markedly higher compared with the expression level in the sham group $(\mathrm{P}<0.05)$, while there was no significant difference between any two groups in the nucleus pulposus (Fig. 6).

\section{Discussion}

To the best of our knowledge, this is the first study to examine the effects of simvastatin on bone loss and IVD degeneration in OVX rats. Based on the analysis of bone mass and biomechanical properties of the vertebrae, in addition to the histological, histomorphometric and immunohistochemical analyses of the IVD, the results of this study demonstrated that osteopenia and IVD degeneration occurred in rats at
6 months after OVX. The results also showed that under the dosage and timescale used in this study, orally administered simvastatin partially preserved the bone mass and biomechanical properties of the lumbar vertebrae. However, there were no protective effects of simvastatin on IVD degeneration in this model.

The OVX rat has been widely used as an estrogen-deficient model for the research study of post-menopausal bone loss $(8-11,13,14,18-20)$. In addition to bone loss and the deterioration of biomechanical properties, IVD degeneration is also observed in this model. This includes histological degeneration shown by VG staining, and histomorphometric findings, such as loss of disc height, thickening of the endplate and an increased ratio of calcified endplate area to total endplate area in the present; all of these changes were similar to those observed in our previous studies (8-10).

The IVD is composed of three distinct tissues: Annulus fibrosus, nucleus pulposus and cartilage endplates (21). Extracellular matrix (ECM) metabolism in the three distinct tissues plays a key role in the integrity and function of the IVD. Col-II and AGG content are crucial to proper disc function. During degeneration, the synthesis of ECM components changes, leading to an increase in the synthesis of col-I and decreased production of AGG (22). In the present study, immunohistochemical analysis revealed decreased expression levels of col-II and AGG, as well as increased expression levels of col-I in the annulus fibrosus and cartilage endplates, confirming the degenerative changes in the ECM. However, contrasting results were found in the nucleus pulposus, which may be explained by the differences of cell type between the different groups. In fact, the histological findings suggest that cell types in the nucleus pulposus changed as IVD degeneration developed, whereby notochordal cells were replaced by chondrocyte-like cells. These different cell types would likely lead to higher expression levels of col-II in the $\mathrm{OVX}+\mathrm{V}$ group compared with the sham group.

Our previous studies demonstrated that alendronate and calcitonin had a dual effect in inhibiting bone loss and reducing IVD degeneration in the OVX rat model $(9,10)$. Simvastatin, a commonly used lipid-lowering drug, has also been shown to be effective for promoting bone formation (12-15), preventing injury-induced IVD degeneration by local injection (16) and promoting chondrogenesis of IVD cells (23). Therefore, it is reasonable to hypothesize that orally administered simvastatin could retard the pathological process of IVD degeneration (24). However, the positive effect of simvastatin was only observed on the vertebrae 


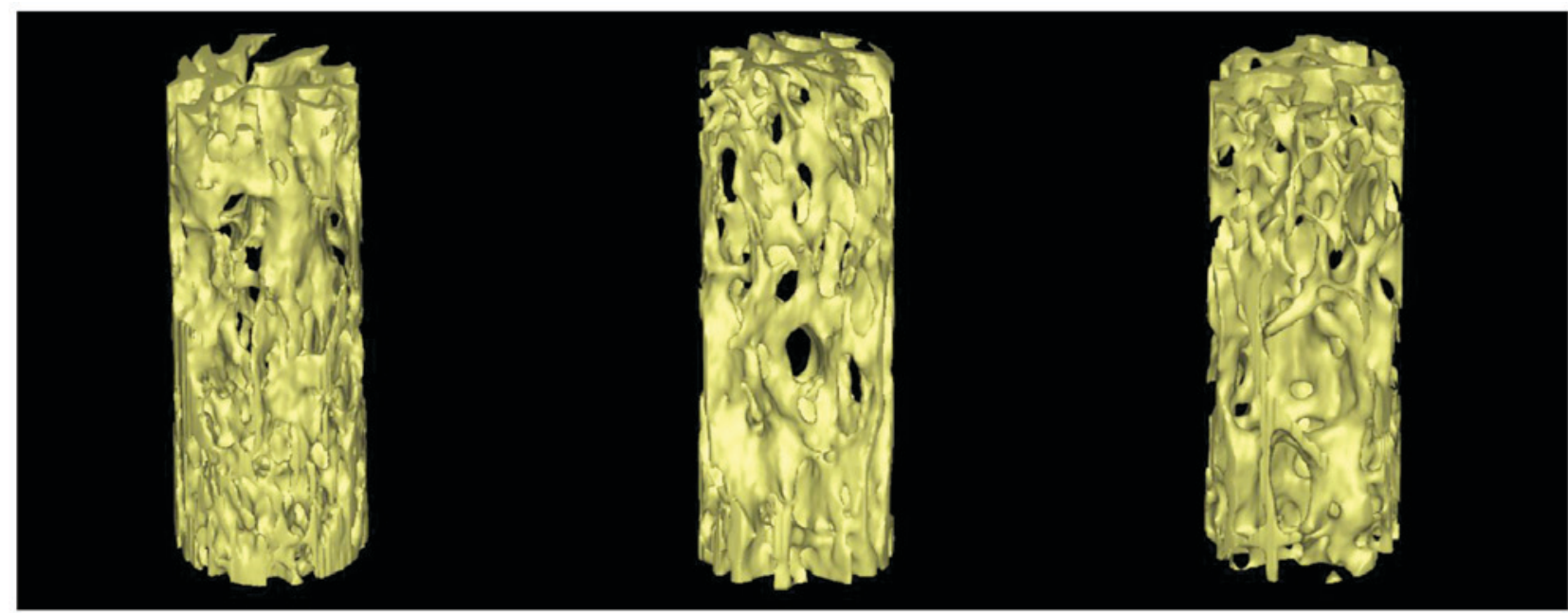

Sham

OVX+V

OVX+SIM

Figure 1. Representative micro-computed tomography images of the volume of interest in the L3 vertebra. OVX, ovariectomized; V, vehicle; SIM, simvastatin.

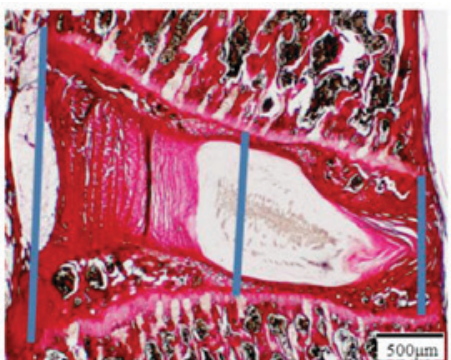

Sham

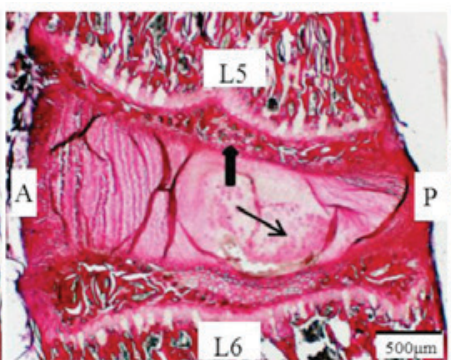

OVX $+\mathrm{V}$

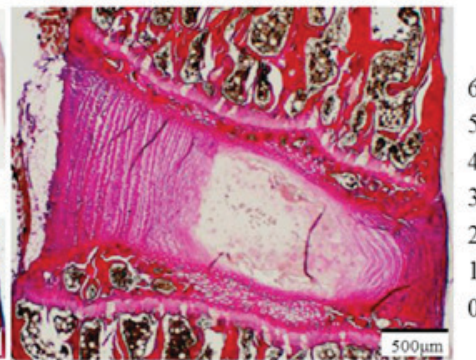

OVX+SIM

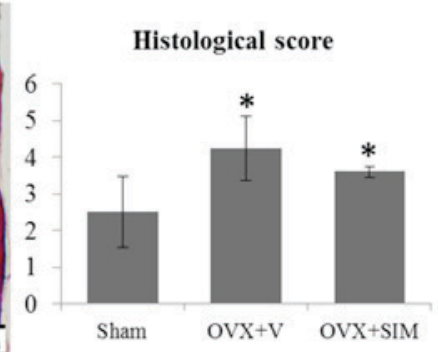

Figure 2. Histological images of the L5-6 segments of the lumbar spine with van Gieson staining. As the black arrows indicate, in comparison with the sham group, in the OVX $+\mathrm{V}$ group, mineralized tissue was more abundant in the deep zone of middle cartilage endplate (thick arrow), and the nucleus pulposus exhibited significant loss of cellularity in notochordal cells and mucoid degeneration (thin arrow). The blue lines mark the three exact locations at which disc height was measured. " $\mathrm{P}<0.05$ vs. sham group. A, anterior side; P, posterior side; OVX, ovariectomized; V, vehicle; SIM, simvastatin.
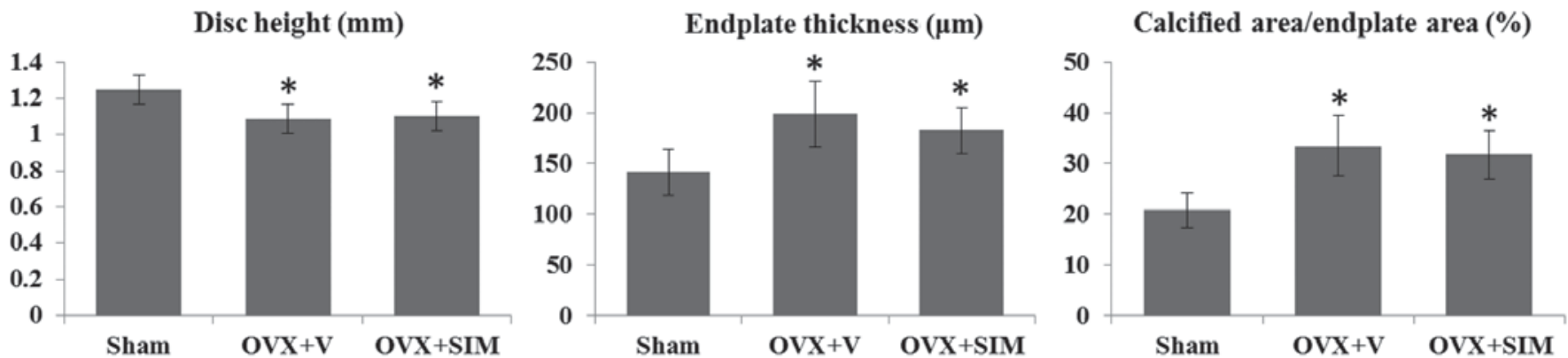

Figure 3. Disc height, endplate thickness and the ratio of calcified area to endplate area of the endplate of the L5-6 disc. "P<0.05 vs. sham group. OVX, ovariectomized; V, vehicle; SIM, simvastatin.

and not on the IVD, which could be attributed to the pharmacokinetic characteristics of the latter and the anatomical characteristics. Orally administered simvastatin is highly lipophilic and undergoes first-pass metabolism, resulting in the bioavailability of this lipophilic statin being very low at $<5 \%$ (25-27). By contrast, as the largest avascular tissue without penetrating blood vessels, the IVD obtains nutrients mostly from and expels waste (lactate) into the adjacent vertebral body via permeable vertebral endplates by diffusion of blood (28); thus, the supply of simvastatin to the IVD would be lower than that to the vertebral body. These factors suggest that the probability of simvastatin reaching therapeutic levels in the IVD is very low following oral administration, which may explain the lack of effect on the IVD in this study. To address this, a much higher dose of oral simvastatin may be required to reach an effective threshold concentration for the inhibition of IVD degeneration. However, it remains unknown whether greater benefit can be attained with higher 


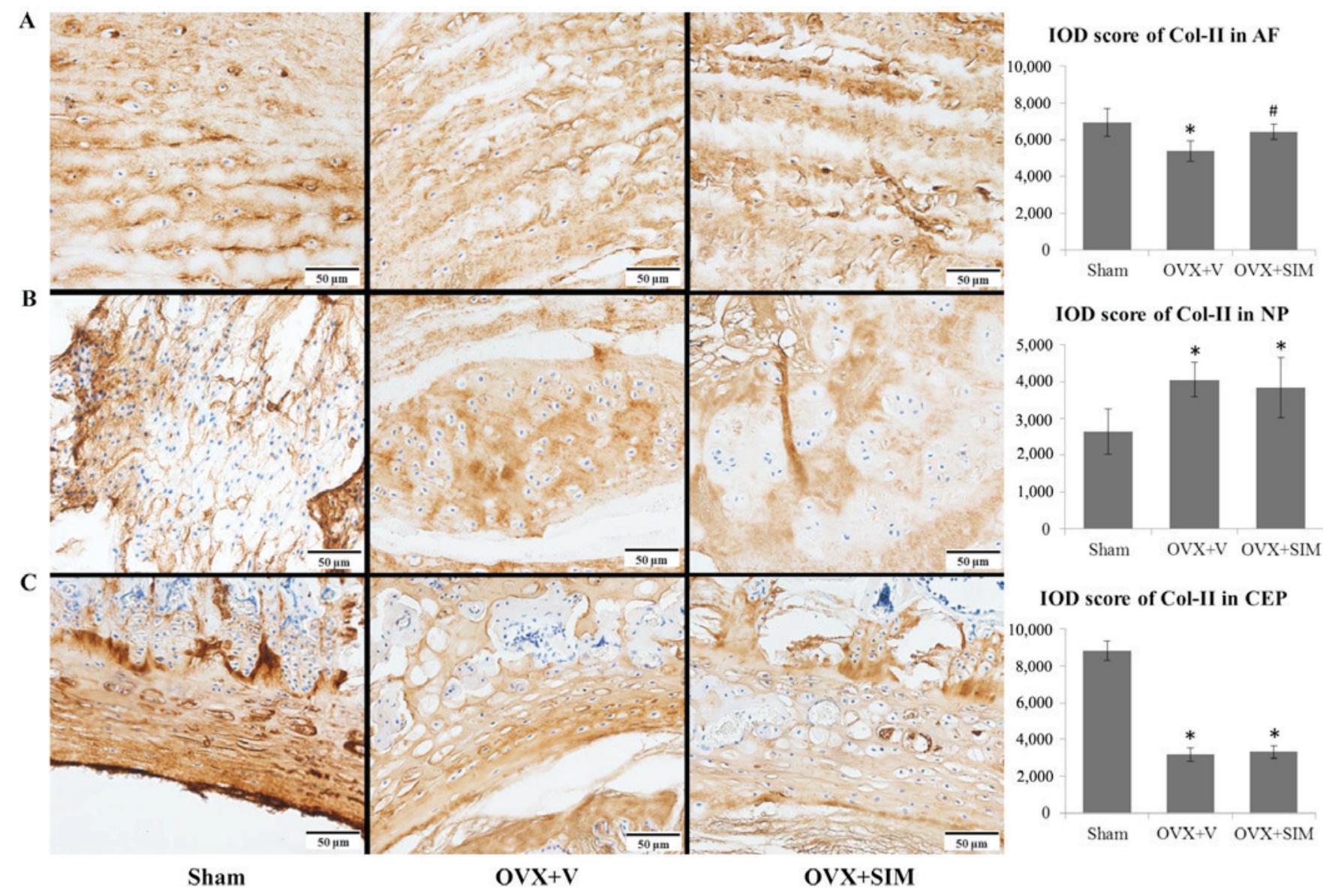

Figure 4. Immunohistochemistry assay results and IOD of Col-II expression in the (A) AF, (B) NP and (C) CEP in the three groups. Immunohistochemical analysis indicated that the protein expression of Col-II in the AF in the OVX + V group was lower than that in the sham and OVX + SIM groups, while in the NP, Col-II expression was significantly lower in the sham group than in the other two groups; inverse results were obtained for the CEP. * $<0.05$ vs. sham group; "P<0.05 vs. OVX + V group. IOD, integrated optical density; Col-II, collagen-II; AF, annulus fibrosis; NP, nucleus pulposus; CEP, cartilage endplate; OVX, ovariectomized; V, vehicle; SIM, simvastatin.

dosages of simvastatin. High-dose statins could even lead to the death of disc cells and should be confirmed to be safe prior to use (16). Further study is also necessary to determine the optimal oral dosage of simvastatin to prevent IVD degeneration. Finally, although there is a lack of direct evidence, simvastatin may be effective in preventing IVD degeneration if administered earlier. In this study, rats received simvastatin at 3 months after OVX was performed, when IVD degeneration had already occurred (10). Future studies should examine whether simvastatin treatment at earlier time points post-OVX is able inhibit IVD degeneration.

It is notable that, in the present study, simvastatin treatment resulted in partial preservation of vertebral BMD and non-significant improvement of trabecular bone volume. However, it remains to be addressed why simvastatin demonstrated a greater benefit on total vertebrae than trabecular bone. It may be hypothesized that the BMD results reflect the effects of simvastatin on the total vertebrae, which was due to the combined effects of simvastatin on both cortical bone and trabecular bone. These factors are additive to each other but may not be significant alone. Furthermore, in comparison with the values from the sham group, the comparable biomechanical properties were unexpected considering the partial preservation of vertebral BMD. The more notable improvement of bone biomechanical properties compared with BMD was also difficult to explain, although similar results have been reported in a previous study (29), where after 3 weeks of simvastatin treatment, the biomechanical parameters of tibias improved but the BMD was not significantly different from that of the control group.

In summary, under the dosage and intervention period used in the present study, orally administered simvastatin partially prevented bone loss and the deterioration of biomechanical properties of lumbar vertebrae, but did not produce a significant preventive effect on the progression of IVD degeneration in OVX rats. Therefore, systemic administration of simvastatin to treat IVD degeneration should be considered with caution. Further study is necessary to exploit the bone- and joint-related ancillary effects of simvastatin by systemic administration, in contrast to local application or tissue-specific non-invasive delivery systems $(30,31)$, before it is finally able to benefit osteoporotic patients with IVD degeneration.

\section{Acknowledgements}

This study was supported by the Natural Science Foundation of Hebei Province (H2013209255) and the Scientific Research Project of Hebei Universities and Colleges (QN20131007). 
A

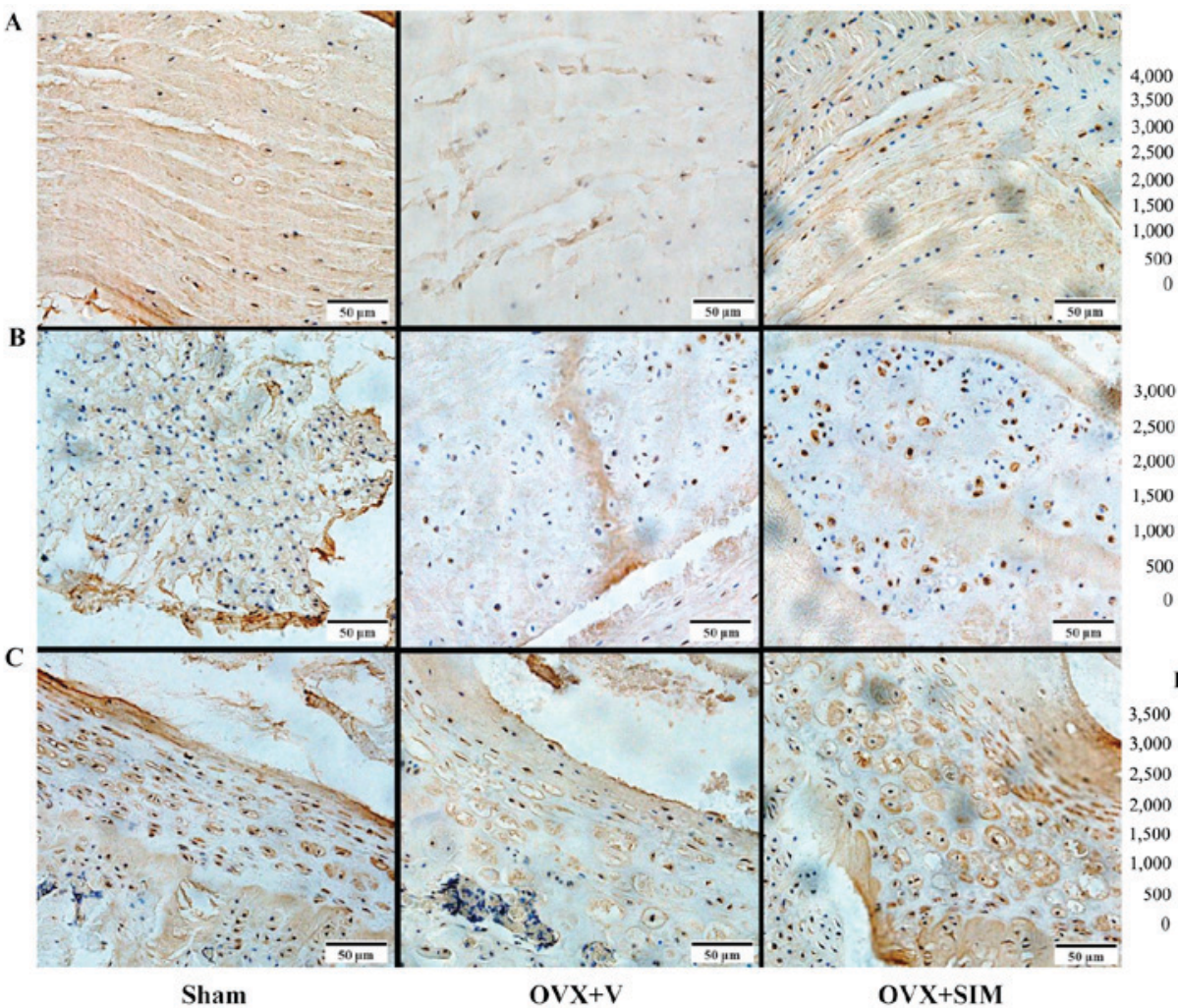

IOD score of AGG in AF

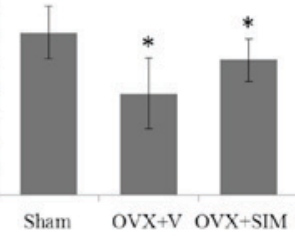

IOD score of AGG in NP

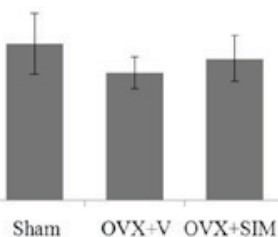

IOD score of AGG in CEP

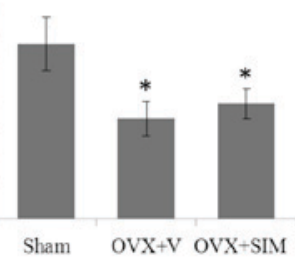

Figure 5. Immunohistochemical assay results and IOD of AGG expression in the (A) AF, (B) NP and (C) CEP in the three groups. Immunohistochemical analysis showed that the protein expression levels of AGG in the AF and CEP in the OVX $+\mathrm{V}$ and OVX + SIM groups were markedly lower than those in the sham group, while no significant difference was found between any two groups in the NP. * $<<0.05$ vs. sham group. IOD, integrated optical density; AGG, aggrecan; AF, annulus fibrosis; NP, nucleus pulposus; CEP, cartilage endplate; OVX, ovariectomized; V, vehicle; SIM, simvastatin.

A
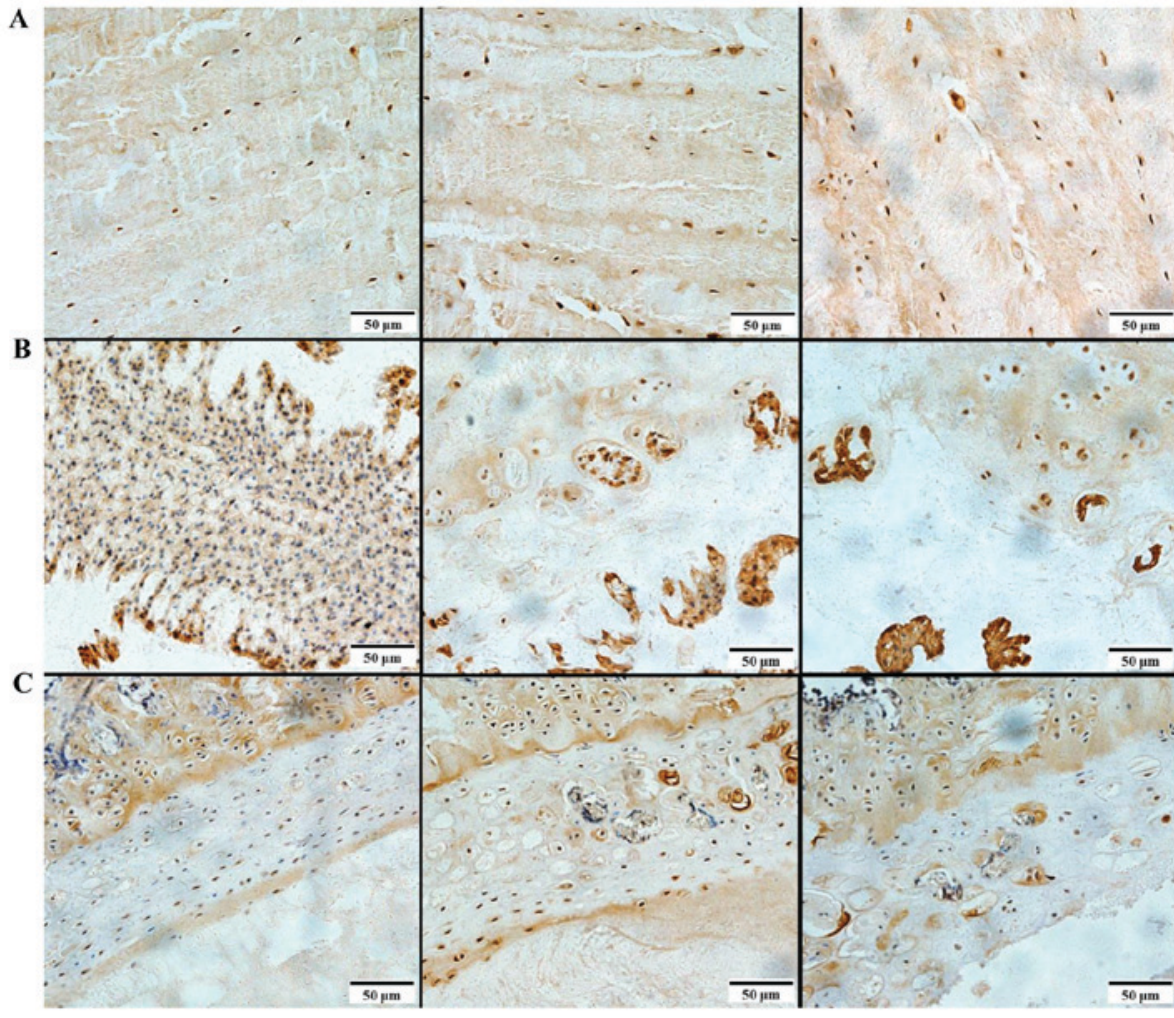

Sham

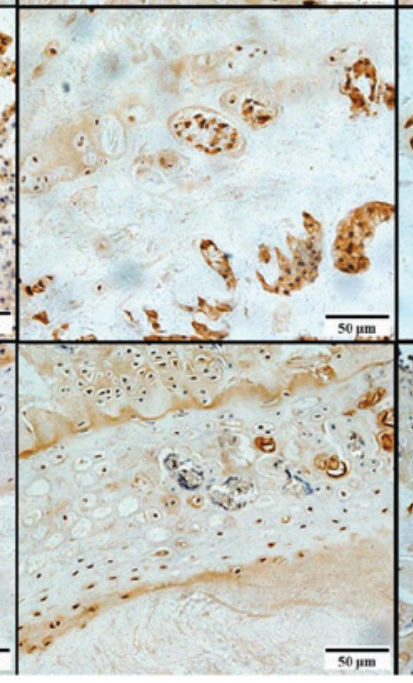

OVX+V

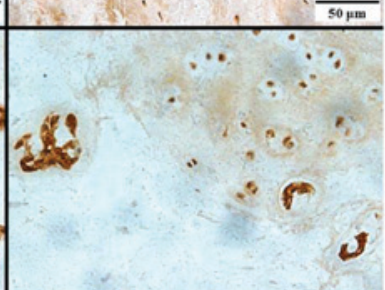

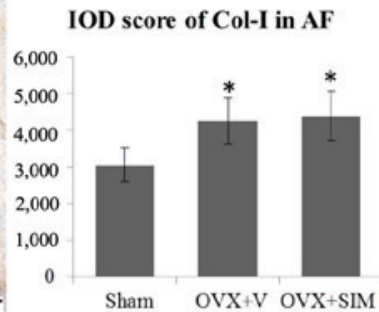

IOD score of Col-I in NP

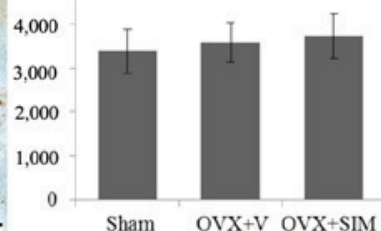

IOD score of Col-I in CEP

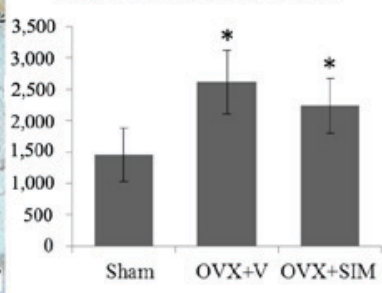

Figure 6. Immunohistochemical assay results and IOD of Col-I expression in the (A) AF, (B) NP and (C) CEP in the three groups. Immunohistochemical analysis showed that the protein expression levels of Col-I in the AF and CEP in the OVX + V and OVX + SIM groups were markedly higher than those in the sham group, while no significant difference was found between any two groups in the NP. ${ }^{*} \mathrm{P}<0.05$ vs. sham group. IOD, integrated optical density; Col-I, collagen-I; AF, annulus fibrosis; NP, nucleus pulposus; CEP, cartilage endplate; OVX, ovariectomized; V, vehicle; SIM, simvastatin 


\section{References}

1. Manek NJ and MacGregor AJ: Epidemiology of back disorders: Prevalence, risk factors, and prognosis. Curr Opin Rheumatol 17: 134-140, 2005.

2. Masuda K and An HS: Prevention of disc degeneration with growth factors. Eur Spine J 15 (Suppl 3): S422-S432, 2006.

3. Andersson GB: Epidemiological features of chronic low-back pain. Lancet 354: 581-585, 1999.

4. Feng C, Liu H, Yang Y, Huang B and Zhou Y: Growth and differentiation factor-5 contributes to the structural and functional maintenance of the intervertebral disc. Cell Physiol Biochem 35: 1-16, 2015.

5. Hadjipavlou AG, Tzermiadianos MN, Bogduk $N$ and Zindrick MR: The pathophysiology of disc degeneration: A critical review. J Bone Joint Surg Br 90: 1261-1270, 2008.

6. Fabreguet I, Fechtenbaum J, Briot K, Paternotte S and Roux C: Lumbar disc degeneration in osteoporotic men: Prevalence and assessment of the relation with presence of vertebral fracture J Rheumatol 40: 1183-1190, 2013.

7. Wang YX and Griffith JF: Effect of menopause on lumbar disc degeneration: Potential etiology. Radiology 257: 318-320, 2010

8. Wang T, Zhang L, Huang C, Cheng AG and Dang GT: Relationship between osteopenia and lumbar intervertebral disc degeneration in ovariectomized rats. Calcif Tissue Int 75 205-213, 2004

9. Luo Y, Zhang L, Wang WY, Hu QF, Song HP, Su YL and Zhang YZ: Alendronate retards the progression of lumbar intervertebral disc degeneration in ovariectomized rats. Bone 55 439-448, 2013.

10. Tian FM, Yang K, Wang WY, Luo Y, Li SY, Song HP, Zhang YZ, Shen Y and Zhang L: Calcitonin suppresses intervertebral disk degeneration and preserves lumbar vertebral bone mineral density and bone strength in ovariectomized rats. Osteoporos Int 26: 2853-2861, 2015.

11. Chuengsamarn S, Rattanamongkoulgul S, Suwanwalaikorn S, Wattanasirichaigoon S and Kaufman L: Effects of statins vs. Non-statin lipid-lowering therapy on bone formation and bone mineral density biomarkers in patients with hyperlipidemia Bone 46: 1011-1015, 2010.

12. Yang N, Cui Y, Tan J, Fu X, Han X, Leng H and Song C: Local injection of a single dose of simvastatin augments osteoporotic bone mass in ovariectomized rats. J Bone Miner Metab 32: 252-260, 2014.

13. Hernández JL, Olmos JM, Romaña G, Martinez J, Castillo J, Yezerska I, Pinedo G and González-Macías J: Bone mineral density in statin users: A population-based analysis from a Spanish cohort. J Bone Miner Metab 32: 184-191, 2014.

14. Tan J, Yang N, Fu X, Cui Y, Guo Q, Ma T, Yin X, Leng H and Song C: Single-dose local simvastatin injection improves implant fixation via increased angiogenesis and bone formation in an ovariectomized rat model. Med Sci Monit 21: 1428-1439, 2015

15. Oxlund $\mathrm{H}$ and Andreassen TT: Simvastatin treatment partially prevents ovariectomy-induced bone loss while increasing cortical bone formation. Bone 34: 609-618, 2004.

16. Than KD, Rahman SU, Wang L, Khan A, Kyere KA, Than TT, Miyata Y, Park YS, La Marca F, Kim HM, et al: Intradiscal injection of simvastatin results in radiologic, histologic, and genetic evidence of disc regeneration in a rat model of degenerative disc disease. Spine J 14: 1017-1028, 2014.
17. Mosekilde L, Danielsen CC and Knudsen UB: The effect of aging and ovariectomy on the vertebral bone mass and biomechanical properties of mature rats. Bone 14: 1-6, 1993.

18. Sehmisch S, Galal R, Kolios L, Tezval M, Dullin C, Zimmer S, Stuermer KM and Stuermer EK: Effects of low-magnitude, high-frequency mechanical stimulation in the rat osteopenia model. Osteoporos Int 20: 1999-2008, 2009.

19. Khan K, Sharan K, Swarnkar G, Chakravarti B, Mittal M, Barbhuyan TK, China SP, Khan MP, Nagar GK, Yadav D, et al: Positive skeletal effects of cladrin, a naturally occurring dimethoxydaidzein, in osteopenic rats that were maintained after treatment discontinuation. Osteoporos Int 24: 1455-1470, 2013.

20. Oliver RA, Yu Y, Yee G, Low AK, Diwan AD and Walsh WR: Poor histological healing of a femoral fracture following 12 months of oestrogen deficiency in rats. Osteoporos Int 24: 2581-2589, 2013.

21. Roberts S, Evans H, Trivedi J and Menage J: Histology and pathology of the human intervertebral disc. J Bone Joint Surg Am 88 (Suppl 2): S10-S14, 2006.

22. Le Maitre CL, Pockert A, Buttle DJ, Freemont AJ and Hoyland JA: Matrix synthesis and degradation in human intervertebral disc degeneration. Biochem Soc Trans 35: 652-655, 2007.

23. Zhang $\mathrm{H}$ and Lin CY: Simvastatin stimulates chondrogenic phenotype of intervertebral disc cells partially through BMP-2 pathway. Spine (Phila Pa 1976) 33: E525-E531, 2008.

24. Shi S, Wang C, Yuan W, Wang X and Zhou X: Potential prevention: Orally administered statins may retard the pathologic process of disc degeneration. Med Hypotheses 76: 125-127, 2011.

25. Corsino A, Bellosta S, Baetta R, Fumagalli R, Paoletti R and Bernini F: New insights into the pharmacodynamic and pharmacokinetic properties of statins. Pharmacol Ther 84: 413-428, 1999.

26. Reinoso RF, SánchezNavarro A, García MJ and Prous JR Preclinical pharmacokinetics of statins. Methods Find Exp Clin Pharmacol 24: 593-613, 2002.

27. William D and Feely J: Pharmacokinetic-pharmacodynamic drug interaction with HMG-CoA reductase inhibitors. Clin Pharmacokinet 41: 343-370, 2002.

28. Soukane DM, Shirazi-Adl A and Urban JP: Computation of coupled diffusion of oxygen, glucose and lactic acid in an intervertebral disc. J Biomech 40: 2645-2654, 2007.

29. Kaleağasıoğlu F, Olcay E and Olgaç V: Statin-induced calcific Achilles tendinopathy in rats: Comparison of biomechanical and histopathological effects of simvastatin, atorvastatin and rosuvastatin. Knee Surg Sports Traumatol Arthrosc, 2015 (Epub ahead of print).

30. Miyazawa A, Matsuno T, Asano K, Tabata Y and Satoh T: Controlled release of simvastatin from biodegradable hydrogels promotes odontoblastic differentiation. Dent Mater J 34: 466-474, 2015.

31. Oryan A, Kamali A and Moshiri A: Potential mechanisms and applications of statins on osteogenesis: Current modalities, conflicts and future directions. J Control Release 215: 12-24, 2015. 